\title{
Clozapine in a patient with treatment-resistant schizophrenia and hypertrophic cardiomyopathy: a case report
}

\author{
Asiel Yair Adan Sanchez, Jessica J. Foster, Carla M. Plymen and Sukhi Shergill
}

\section{Background}

There is currently limited experience in the initiation and maintenance of clozapine for treatment-resistant psychosis in adults with established structural heart disease. These complex patients require close supervision and liaison between colleagues. Here we present the successful experience of treating one such patient within our service and describe a monitoring plan to ensure that these treatments can be provided both safely and effectively.

\section{Case presentation}

A 36-year-old man with treatment-resistant schizophrenia and known hypertrophic cardiomyopathy (HCM) was admitted to a specialist unit for a trial of clozapine. His psychiatric illness was characterised by multimodal hallucinations and delusions combined with low mood and poor motivation. The diagnosis of HCM was made 3 years previously following a routine electrocardiogram (ECG), and he had remained asymptomatic throughout this time; there were concerns about the risk of initiating clozapine given his pre-existing cardiac condition. Baseline investigations were performed as per local guidelines prior to commencing clozapine; these were within normal limits other than a mildly raised troponin level of $54 \mathrm{ng} / \mathrm{L}$ (normal $<16 \mathrm{ng} / \mathrm{L}$ ), which was attributed to the HCM. In addition, baseline transthoracic echocardiography (TTE) was performed which showed no change in the structural heart disease in comparison with previous TTEs.

Clozapine was started at $12.5 \mathrm{mg}$ daily and up-titrated to $150 \mathrm{mg}$ twice daily over 14 days as per our institute's guidelines. The patient was monitored with regular testing of troponins, inflammatory markers and ECG. On day 18 , the troponin level increased to $1371 \mathrm{ng} / \mathrm{L}$. Creatine kinase and inflammatory markers remained stable. No changes in ECG or TTE were noted and the patient remained clinically asymptomatic.

Cardiology opinion was sought and reported that the finding of an isolated elevated troponin was likely to reflect a "troponin leak' in the context of increased cardiac muscle mass associated with HCM. In the absence of any clinical compromise, it was not felt to be of concern. Clozapine was continued with good effect on mental state. Troponin levels gradually reduced and the patient remained well.

\section{Conclusions}

While multiple cases of clozapine-induced cardiotoxicity have been reported in the literature, its implications for pre-existing structural disease are unclear. This case report suggests that clozapine can be safely introduced in pre-existing HCM, explores strategies for monitoring and highlights the importance of liaising with experienced cardiologists.

\section{Declaration of interest}

None.

\section{Copyright and usage}

(C) The Royal College of Psychiatrists 2016. This is an open access article distributed under the terms of the Creative Commons Non-Commercial, No Derivatives (CC BY-NC-ND) license.
Clozapine is considered the antipsychotic agent of choice in the treatment of treatment-resistant schizophrenia, offering reduction in core positive symptoms for $50 \%$ of patients. ${ }^{1}$ These include hallucinations, delusions and thought disorder, as well as suicidality. ${ }^{2,3}$ However, clozapine use is associated with a risk of lifethreatening agranulocytosis for $1-2 \%$ of patients ${ }^{4}$ and cardiotoxicity for $0.1-1.2 \%$ of patients. ${ }^{5-7}$ As such, treatment with clozapine is recommended for patients that have failed to respond to treatment with two antipsychotic medications given at appropriate doses and for a suitable duration.

As stated above, clozapine is associated with cardiotoxicity; specifically, clozapine use is often linked with the development of a sinus tachycardia and hypotension, the long-term significance of which as yet remains unclear. Of greater concern is the less common development of an acute myocarditis and/or pericarditis and a more chronic dilated cardiomyopathy. ${ }^{5,6}$ These conditions carry significant comorbidity and mortality. The vast majority of available literature examining the cardiac effects of clozapine involves studies in those with previously normal hearts; there is very little data to guide the safe use of clozapine in patients with established structural heart disease.

Hypertrophic cardiomyopathy (HCM) is the commonest inherited form of cardiovascular disease with an estimated prevalence of 1 in 500. It shows autosomal dominant transmission, and increased left ventricular wall mass is pathognomonic of the disease. Although the majority of those with phenotypic disease will have a benign course, HCM is associated with sudden cardiac death, syncope, stroke and heart failure; ${ }^{8}$ findings of investigations, including resting electrocardiogram (ECG) and cardiac enzymes, as well as transthoracic echocardiography (TTE) are usually markedly abnormal.

This report presents our experience in initiating clozapine in a young man with treatment-resistant schizophrenia and concomitant complex cardiac disease and suggests a monitoring plan that could be used with similar patients with cardiac comorbidity.

\section{Case presentation}

The patient was a 36-year-old British male of Iranian decent with a diagnosis of treatment-resistant schizophrenia. He was single, unemployed and living with his family. He was referred for a trial of clozapine in the context of an established diagnosis of HCM (Fig. 1). At the time of admission, he presented with multimodal hallucinations, thought disorder, delusional beliefs and psychosomatic symptoms; he had no cardiovascular symptoms. On admission, he scored 40 on the Psychotic Symptom Rating Scales

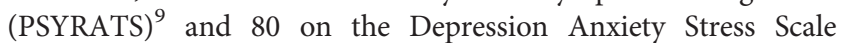
(DASS). ${ }^{10} \mathrm{He}$ had previously trialled a number of antipsychotic 
(a)
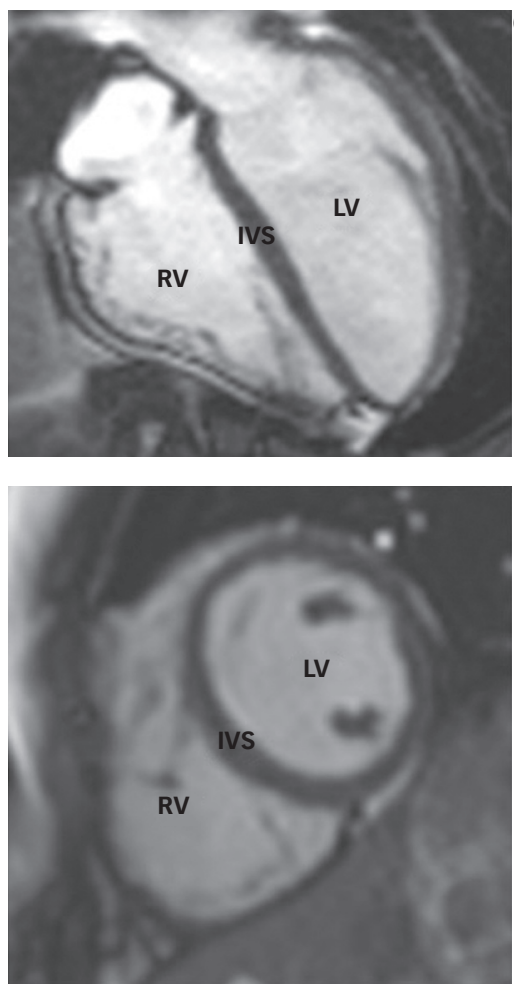
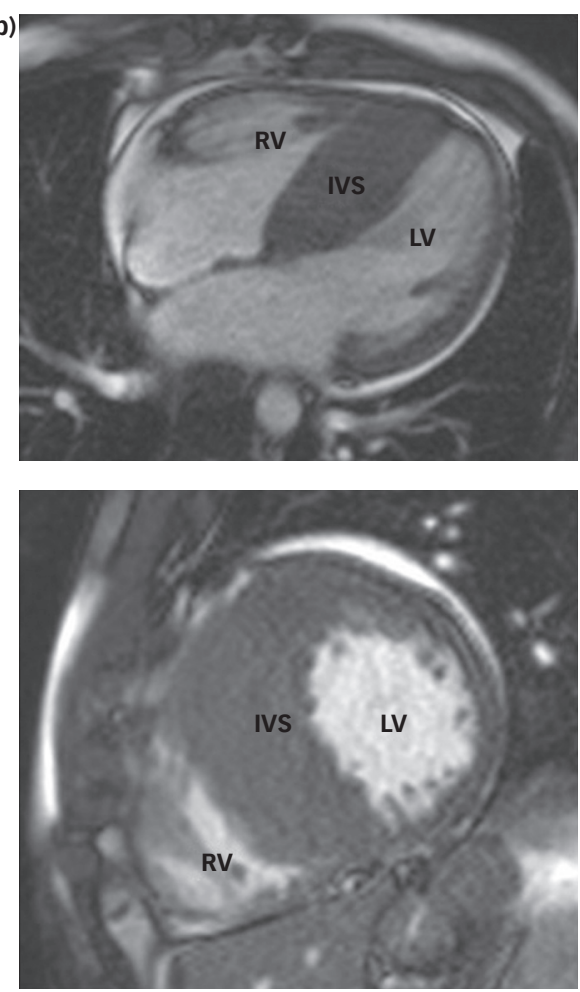

Fig. 1 Cardiac magnetic resonance images of a normal heart (a) and a heart demonstrating hypertrophic cardiomyopathy (b). Note the significant increase in muscle mass at the intraventricular septum (IVS) in the abnormal heart (b). LV - left ventricle; RV - right ventricle.

agents with limited improvement, including aripiprazole, paliperidone, amisulpride and risperidone. Flupentixol and olanzapine had been moderately successful in treating his positive symptoms, but had to be stopped because of adverse motor effects. At the time of admission, he was on amisulpride $800 \mathrm{mg}$ and sertraline $100 \mathrm{mg}$ for low mood.

Relevant past medical history includes the diagnosis of HCM, made 3 years prior following the finding of an abnormal ECG. Further diagnostic investigations had included TTE, 24-h Holter monitoring and cardiovascular magnetic resonance imaging (CMR). During the 3-year follow-up period, the patient had been asymptomatic and was not felt to be at significant risk of sudden cardiac death (Table 1). He was taking bisoprolol $7.5 \mathrm{mg}$ daily to treat hypertension - this being the agent of choice in those with HCM to help ameliorate the potential risk of arrhythmic demise and outflow obstruction associated with HCM. Other cardiovascular risk factors include a 17 pack/year smoking history and hypercholesterolaemia controlled with atorvastatin $40 \mathrm{mg}$ daily.

The patient leads a moderately active lifestyle without limitation.

Table 1 Major clinical risk factors for sudden cardiac death in
HCM $^{a}$
Age (younger patients may be more at risk)
Non-sustained VT
LV hypertrophy $>30 \mathrm{~mm}$
Syncope
Family history of sudden death
Left atrial diameter
LV outflow tract obstruction
Blunted exercise BP response
a. From Elliot et al ${ }^{11}$
VT, ventricular tachycardia; LV, left ventricle; BP, blood pressure

\section{Investigations and initiation of clozapine}

On arrival to our unit, the patient had a heart rate of 76 beats per minute (bpm) in sinus rhythm. A baseline ECG (Fig. 2) was carried out to monitor for any changes once clozapine was started. He was stable and asymptomatic at the time. Body mass index was high at $32 \mathrm{~kg} / \mathrm{m}^{2}$. He was hypertensive at $145 / 90 \mathrm{mmHg}$. Baseline cardiac biomarkers showed a troponin of $54 \mathrm{ng} / \mathrm{L}$ (normal $<16 \mathrm{ng} / \mathrm{L}$ ) and creatine kinase of $224 \mathrm{IU} / \mathrm{L}$ (normal <150 IU/L).

Cardiology advice was sought given the history of HCM and abnormal findings. The cardiology opinion concluded that the abnormalities in the cardiac biomarkers represented the patient's own normal baseline levels - given the context of his Iranian ethnicity ${ }^{12}$ and his increased cardiac muscle mass - and that it was therefore reasonable to initiate clozapine treatment. Clozapine was therefore started at $12.5 \mathrm{mg}$ alongside his regular bisoprolol, which was hoped to help reduce the risk of tachycardia. Clozapine was then titrated up to $150 \mathrm{mg}$ twice daily with regular monitoring. The patient was monitored through weekly troponin, creatine kinase and clozapine levels, as well as ECG every 3 weeks.

Figure 3 shows that during weeks $4-5$ of clozapine uptitration, there was a spike in the troponin levels. At this point, the patient remained clinically well and was haemodynamically stable with a heart rate of $90 \mathrm{bpm}$ and a blood pressure of $140 / 80 \mathrm{mmHg}$. Consultation with Cardiology reported that the spike represented a minor troponin leak which, in the absence of any other clinical changes, was not clinically significant. The advice was that we continue with up-titration and with continued blood testing. Figure 3 shows resolution of this event, and regular serum clozapine levels were also noted.

After 3 months of therapy, the patient remained well with a significant reduction in the level of his hallucinations, a cessation of his thought disorder, and decreases in the intensity of his delusional beliefs and psychosomatic symptoms. His scores on the PSYRATS reduced from 40 to 28 and from 80 to 42 on the DASS. 


$\begin{array}{lrrl}\text { Vent. rate } & 90 & \text { BPM } & \text { Normal sinus rhythm } \\ \text { PR interval } & 144 & \text { ms } & \text { Marked ST abnormality, possible inferolateral subendocardial injury } \\ \text { QRS duration } & 92 & \text { ms } & \text { Abnormal ECG } \\ \text { QT/QTC } & 370 / 452 & \text { ms } & \text { No previous ECGs available } \\ \text { P-R-T axes } & 6035 & 216 & \end{array}$

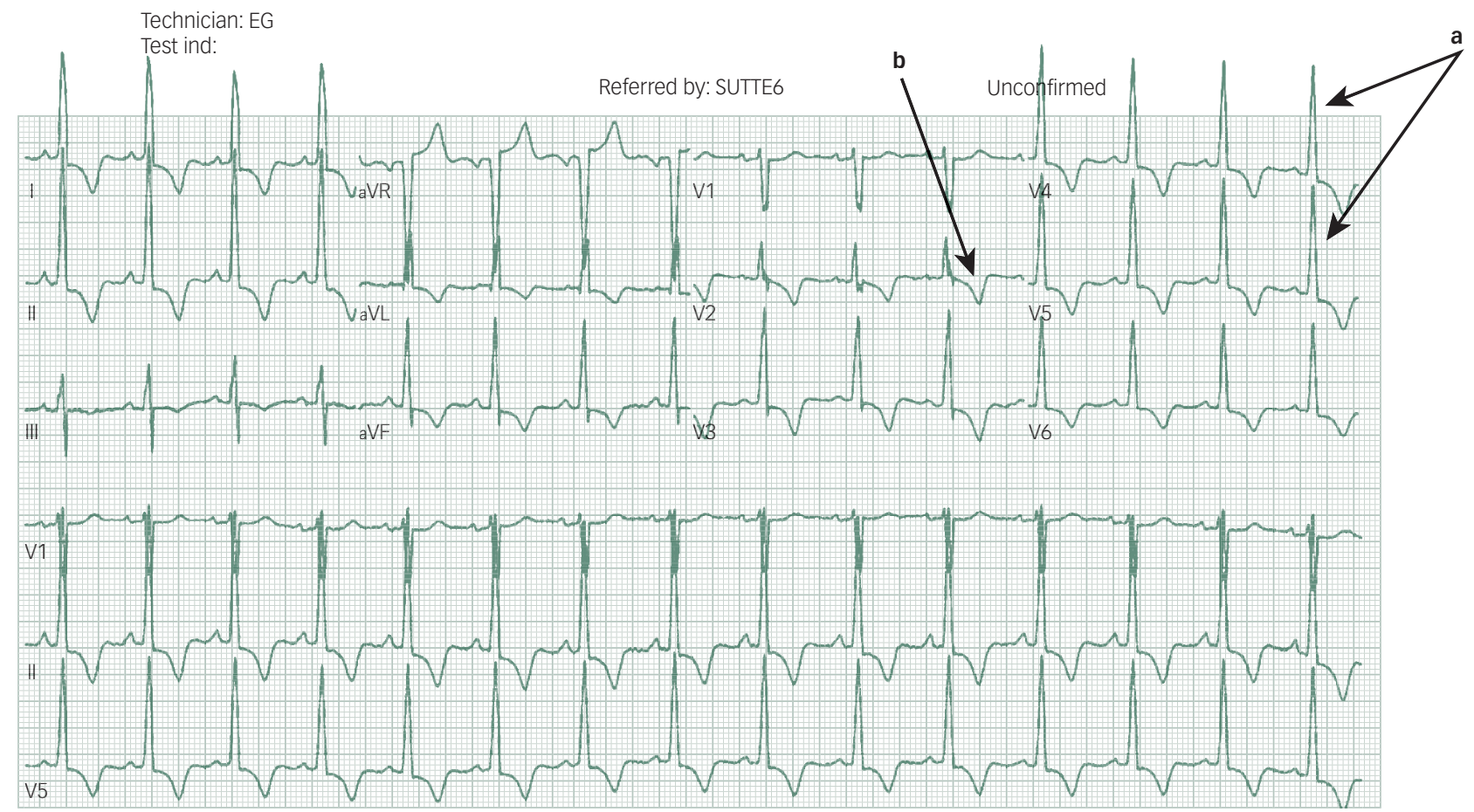

Fig. 2 Electrocardiogram (ECG) showing sinus rhythm with a left ventricular hypertrophy pattern (a) with deep T-wave inversion (b) throughout typically seen in hypertrophic cardiomyopathy.

He returned to a stable social functioning, with minor residual symptoms and increased levels of engagement and motivation. There has been no evidence of worsening of his cardiomyopathy, either clinically or with subsequent investigation. The patient has led a moderately active lifestyle, engaged well with other aspects of therapy and showed increased interest in his care.

Initiating clozapine therapy in patients with pre-existing HCM requires liaison with Cardiology for expert opinion, as well as close monitoring for any evidence of cardiac decompensation. There are currently no evidence-based guidelines for initiating clozapine in such patients; nonetheless, we show that clozapine can be initiated safely in patients with HCM with careful monitoring and titration.

In our case, despite evidence of structural cardiac disease, the patient was deemed to be at low risk for sudden cardiac death, without obstructive disease and with normal cardiac function. Clozapine was commenced at $12.5 \mathrm{mg}$ and titrated up to $150 \mathrm{mg}$ b.d. as per our own institution's guidelines; this included regular monitoring with ECG and blood testing. Baseline abnormalities required close liaison with our Cardiology colleague; however, the patient's course was uneventful and the treatment successful.

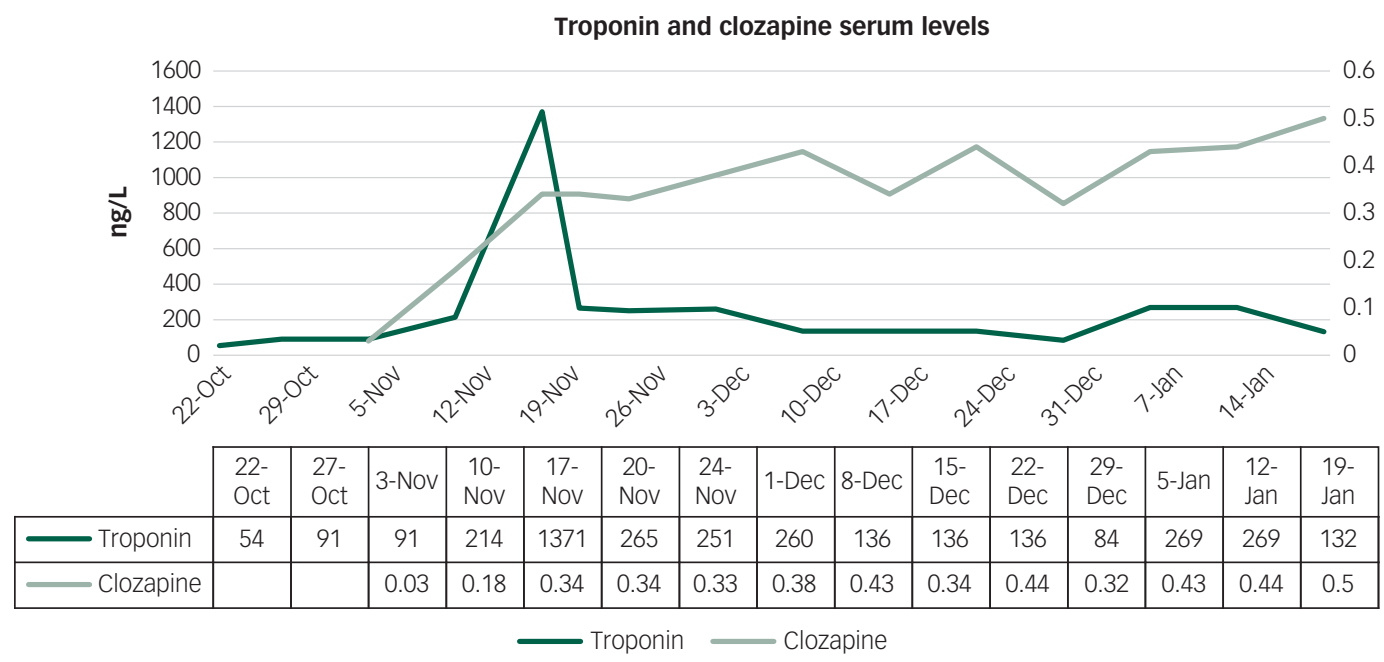

Fig. 3 Graph showing serum levels of both troponin and clozapine during starting and up-titration. 


\begin{tabular}{|c|c|}
\hline meline & Investigations \\
\hline At baseline & Temperature, CRP, troponin, ECG, TTE \\
\hline Daily & Temperature, heart rate \\
\hline 24-h post dose increase & CRP, troponin, ECG \\
\hline $\begin{array}{l}\text { In the event of ongoing fever or } \\
\text { abnormal CRP/troponin }\end{array}$ & Temperature (CRP, troponin) ECG, TTE \\
\hline 6 weeks post final dose increase & Temperature, CRP, troponin, ECG, TTE \\
\hline
\end{tabular}

Despite its efficacy, cardiac side-effects of clozapine can generate concerns about its use in patients with pre-existing cardiovascular disease. Common side-effects include orthostatic hypotension, reported in up to $75 \%$ of treated patients, ${ }^{13}$ in addition to a welldocumented, benign tachycardia seen in up to $25 \%$ of patients. ${ }^{14}$

Clozapine is also associated with more severe forms of cardiac toxicity, most notably myocarditis, ${ }^{5,7}$ dilated cardiomyopathy ${ }^{7}$ and pericarditis. ${ }^{7}$ Development of any of these conditions requires cessation of the causative agent but is often reversible with appropriate treatment. Acute myocarditis can be life-threatening however, and in some, it may not be reversible, leading to significantly increased long-term morbidity and mortality. Currently, there are no established risk factors by which to identify those who may be at increased risk of developing these significant side-effects, and as such, patients starting clozapine are subjected to regular investigations to monitor for any change. It remains unknown whether those with preexisting cardiac disease are at increased risk.

In our patient, a mildly increased baseline troponin was felt to relate to the increased muscle mass of the left ventricle seen in $\mathrm{HCM}$. Troponin is a more specific myocardial marker than creatine kinase, and the brief elevation during the course of clozapine uptitration probably represents a very mild, self-limiting myocarditis. The underlying mechanism of cardiotoxicity by clozapine remains unclear. Several different mechanisms have been proposed, and it is likely that a combination of these is causative in susceptible individuals. It remains to be determined whether the pathophysiological processes at play in HCM, itself a condition with a wide genotypic and phenotypic presentation, pose a greater short- and long-term risk for those concurrently treated with clozapine.

It is interesting to briefly mention the well-documented cardiotoxic effects of chemotherapeutic agents. Anthracyclines and HER2 antagonists are associated with the development of both acute and late onset cardiomyopathy. ${ }^{15}$ Several different modes of action are thought to underlie these, some of which have also been described in animal models of clozapine cardiotoxicity. ${ }^{16}$ It may well be that a similar mechanism of action is attributable to all these agents, and as such, it is very interesting to also note that early treatment with angiotensin-converting enzyme (ACE) inhibitors (standard prognostic anti-heart failure medication) results in a protective effect against the development of cardiotoxicity in both animal models receiving clozapine ${ }^{17}$ and humans receiving chemotherapy. ${ }^{18}$ A new subspecialty has emerged, cardio-oncology, which facilitates best research, investigation and management in order to optimise the treatment and outcomes of those patients with cardiotoxic effects of chemotherapy. One pragmatic suggestion is the need for an analogous 'cardiopsychiatric' team involving, but not limited to, specialists in psychiatry and cardiology. Such a collaboration would provide the most comprehensive care, given the complexity of managing both pre-existing and iatrogenic cardiac disease because of antipsychotic medication. To that end, Table 2 sets out our suggested regime for the monitoring of cardiotoxicity in patients undergoing clozapine initiation and up-titration with known structural heart disease.
Asiel Yair Adan Sanchez, BBMed, Melbourne Medical School, Austin Hospital, The University of Melbourne, Melbourne, Australia; Jessica J. Foster, MB BCh, National Psychosis Unit, South London and Maudsley NHS Foundation Trust, London, UK; Carla M. Plymen, MRCP, MD(Res), Division of Cardiology, King's College Hospital NHS Foundation Trust, London, UK; Sukhi Shergill, MBBS, SFHEA, PhD, FRCPsych, National Psychosis Unit, South London and Maudsley NHS Foundation Trust, London, UK; National Institute for Health Research (NIHR) Biomedical Research Centre at South London and Maudsley NHS Foundation Trust, King's College London, London, UK; Institute of Psychiatry, Psychology and Neuroscience, King's College London, London, UK

Correspondence: Sukhi Shergill, Institute of Psychiatry, Psychology and Neuroscience, King's College London, Denmark Hill, London SE5 9RS, UK. Email: sukhi.shergill@kcl.ac.uk First received 10 Aug 2016, final revision 16 Oct 2016, accepted 17 oct 2016

\section{References}

1 Lieberman JA, Saffermna AZ, Pollack S, Szymanski S, Johns C, Howard A, et al. Clinical effects of clozapine in chronic schizophrenia: response to treatment and predictors of outcome. Am J Psychiatry 1994; 151: 1744-52.

2 Kane J, Honigfeld G, singer J, Meltzer H. Clozapine for the treatment resistant schizophrenic. A double-blind comparison with chlorpromazine. Arch Gen Psychiatry 1988; 45: 789-96.

3 Kane JM. Treatment-resistant schizophrenic patients. J Clin Psychiatry 1996; 57 (suppl. 9): 35-40.

4 Alvir JM, Lieberman JA, Safferman AZ, Schwimmer JL, Schaaf JA. Clozapineinduced agranulocytosis: incidence and risk factors in the United States. N Eng/ J Med 1993; 329: 162-7.

5 Alawami M, Wasywich C, Cicovic A, Kenedi C. A systematic review of clozapine induced cardiomyopathy. Int J Cardiol 2014; 176: 315-20.

6 Berardis DD, Serroni N, Campanella D, Olivieri L, Ferri F, Carano A, et al. Update on the adverse effects of clozapine: focus on myocarditis. Curr Drug Safety 2012; 7: $55-62$.

7 Layland JJ, Liew D, Prior DL. Clozapine-induced cardiotoxicity: a clinical update. Med J Aust 2009; 190: 190-2.

8 Maron BJ, Ommen SR, Semsarian C, Spirito P, Olivotto I, Martin S, et al. Hypertrophic cardiomyopathy. JACC 2014; 64: 83-99.

9 Haddock G, McCarron J, Tarrier N, Faragher EB. Scales to measure dimensions of hallucinations and delusions: the psychotic symptom rating scales (PSYRATS). Psychol Med 1999; 29: 879-89.

10 Lovibond PF, Lovidbond SH. The structure of negative emotional states: comparison of the Depression Anxiety Stress Scale (DASS) with the beck depression and anxiety inventories. Behav Res Ther 1955; 33: 335-43.

11 Elliot PM, Anastasakis A, Borger MA, Borggrefe M, Cecchi F, Charron P, et al. 2014 ESC Guidelines on diagnosis and management of hypertrophic cardiomyopathy. Eur Heart J 2014; 35: 2733-79.

12 Wong ET, Cobb C, Umehara MK, Wolff GA, Haywood LJ, Greenberg T, et al. Heterogeneity of serum creatine kinase activity among racial and gender groups of the population. Am J Clin Pathol 1983; 79: 582-6.

13 Testani M Jr. Clozapine-induced orthostatic hypotension treated with fludrocortisone. J Clin Psychiatry 1994; 55: 497-8.

14 Cohen $\mathrm{H}$, Loewenthal $\mathrm{U}$, Matar MA, Kotler M. Reversal of pathologic cardiac parameters after transition from clozapine to olanzapine treatment: a case report. Clin Neuropharmacol 2001; 24: 106-8.

15 Ewer MS, Ewer SM. Cardiotoxicity of anticancer treatments. Nat Rev Cardiol 2015; 12: $547-58$.

16 Abdel-Wahab BA, Metwally ME. Clozapine-induced cardiotoxicity: role of oxidative stress, tumour necrosis factor alpha and NF-kb. Cardiovasc Toxicol 2015; 15 $355-65$.

17 Abdel-Wahab BA, Metwally ME, El-khawanki MM, Hashim AM. Protective effect of captopril against clozapine-induced myocarditis in rats: role of oxidative stress, proinflammatory cytokines and DNA damage. Chem Biol Interact 2014; 216: 43-52.

18 Cardinale D, Colombo A, Sandri MT, Lamantia G, Colombo N, Civelli M, et al. Prevention of high-dose chemotherapy-induced cardiotoxicity in high-risk patients by angiotensin-converting enzyme inhibition. Circulation 2006; 114: 2474-81. 